\title{
INFLUENCE OF THE THICKNESS OF ENAMEL LAYER ON OVERALL COLOR OF COMPOSITE RESTORATION USING DIFFERENT POLYCHROMATIC COMPOSITE RESINS
}

\author{
Layal Jbara*, Mona Ghoneim** and Hala Ragab ${ }^{* * *}$
}

\begin{abstract}
The purpose of this study was to calculate and study the color components (Chroma and Value) and translucency of resin composites at different thicknesses.

Method: resin composites shade A1 were used in this study; Filtek Z350 XT 3M (FT), Harmonize Kerr (HK), and Empress Direct Ivoclar Vivadent (ED). All samples were fabricated with same dentin thickness of $2 \mathrm{~mm}$, while the enamel samples were prepared in two thickness; $0.5 \mathrm{~mm}$ and $1 \mathrm{~mm}$. Transparency parameter evaluation was done by measuring the CIELAB parameters of each sample once against white background and once against black. $\triangle \mathrm{E}$ was used to evaluate the change in color of composite resin at different thicknesses. One-way ANOVA and Tukey HSD test were used to analyze the descriptive statistics. Pearson correlation coefficient was used to conclude a relation between thickness and chroma; and thickness and value.
\end{abstract}

Results: There was a significant difference in translucency in all composite groups at enamel thickness 0.5 and $1 \mathrm{~mm}$. $\Delta \mathrm{E}$ showed significant difference when comparing group FT with $\mathrm{ED}$ and HK, but no significant difference when comparing groups ED and HK. As for chroma, groups ED and FT showed a decrease in chroma as thickness increased and the results were significant at $(\mathrm{p}<0.05)$, while groups HK showed increase in chroma but the results were insignificant.

Conclusion: As thickness increases the translucency and chroma of the composite resin decreases.

\section{INTRODUCTION}

In today's society the importance of physical appearance has grown tremendously. A pleasing smile is a fundamental prerequisite for professional and social achievement. With this comes the increasing trend of composite veneer among the young generation for its low cost and minimal invasive approach. The wide selection of shades and opacities of composite resin provide

\footnotetext{
* MSc student, Operative and Esthetic dentistry, Faculty of Dentistry, Beirut Arab University, Lebanon.

** Associate Professor of restorative dentistry, faculty of dentistry, Alexandria university, Egypt.

*** Professor of Operative and Esthetic dentistry, Division Director, Department of Restorative Sciences, Faculty of

Dentistry, Beirut Arab University, Lebanon.
} 
practitioners the opportunity to mimic the natural tooth appearance while using different shades and opacities in a single restoration. In order to achieve identical anatomy and optical appearance to natural teeth, layering techniques are essential. ${ }^{2}$

The communication and collaboration of enamel, dentin and pulp with light gives us the tooth color. Each subject has a different characteristic and arrangement; therefore, they reveal different light wave characteristics ${ }^{3}$.

Natural tooth enamel and dentin differ in their optical properties. Enamel has a higher transmission of light than dentin and is usually achromatic but may give a yellowish to white or greyish to white aspect. Dentin is less translucent and its chroma increases over the years. When choosing shades, the color is predominantly determined by the dentin, enamel contributes in the form of scattering of wavelengths in the blue range. ${ }^{3,4,5}$

Refractive index refers to the propagation of light or any other radiation through an optical medium. It is represented by a ratio between speed of light in vacuum and speed of light in the optical medium. Dental tissues and restorative materials have different refractive indices ${ }^{5}$ Therefore, when restoring teeth, the restoration should not be placed in the same thickness as natural tissues or else optical integration will not be successful.

Optical properties of translucency are greatly affected by the thickness, as thickness decreases the translucency of the resin increases greatly. Translucency parameter is a vital parameter used to assess and evaluate the translucency of resin composite, it is done by measuring the color of the same specimen but with white and then black backings . ${ }^{6,78}$

The aim of this study was to evaluate the influence of enamel thickness on the overall color of restoration and translucency in different brands of resin composite with a fixed dentin thickness.

The null hypothesis tested is that an increase in thickness of enamel will not have any significant change on the chroma and translucency of the polychromatic composite resin

\section{MATERIALS AND METHODS}

The purpose of this in-vitro study is to evaluate the color change between different enamel thicknesses with fixed dentin thickness of $2 \mathrm{~mm}$. The comparison was made between three commercially available resin composite brands Ivoclar Vivadent Empress Direct, Kerr Harmonize and 3m Filtek z350 XT. The shade selected was A1 for both dentin and enamel in all composite brands. The specimens were prepared in the laboratories at Beirut Arab University.

\section{Sample}

Thicknesses of the composite for all samples must be of identical measurement, therefore a custom made Teflon split molds were fabricated to produce disk like specimens with diameter of $8 \mathrm{~mm}$ and thickness of $2 \mathrm{~mm}, 2.5 \mathrm{~mm}$, and $3 \mathrm{~mm}$ ???? A total of 6 specimens for each brand and thickness were fabricated by placing the mold on a glass slab. After the composite was condensed in the mold a celluloid strip was placed on top and covered by another glass slab to insure a flat top and base finger pressure was applied. ${ }^{3}$ A LED curing light (bluephase, style 20i, ivoclar/vivadent) was used to light cure the specimens for 40 seconds and $1 \mathrm{~mm}$ away from each specimen top and bottom.

TABLE (1) Composite Material used in this study

\begin{tabular}{|c|c|c|c|c|}
\hline Code & Product & Shade & $\begin{array}{l}\text { Lot } \\
\text { number }\end{array}$ & Manufacturer \\
\hline \multirow{2}{*}{ FT } & \multirow{2}{*}{$\begin{array}{l}\text { Filtek } \\
\text { Z350 XT }\end{array}$} & A1 dentin & $?$ & \multirow{2}{*}{$3 \mathrm{M}$} \\
\hline & & A1 enamel & $? ?$ & \\
\hline \multirow{2}{*}{ ED } & \multirow{2}{*}{$\begin{array}{l}\text { Empress } \\
\text { direct }\end{array}$} & A1 dentin & $?$ & \multirow{2}{*}{$\begin{array}{l}\text { Ivoclar, } \\
\text { vivadent }\end{array}$} \\
\hline & & A1 enamel & $?$ & \\
\hline \multirow{2}{*}{ HK } & \multirow{2}{*}{ Harmonize } & A1 dentin & $?$ & \multirow{2}{*}{ Kerr } \\
\hline & & A1 enamel & $?$ & \\
\hline
\end{tabular}




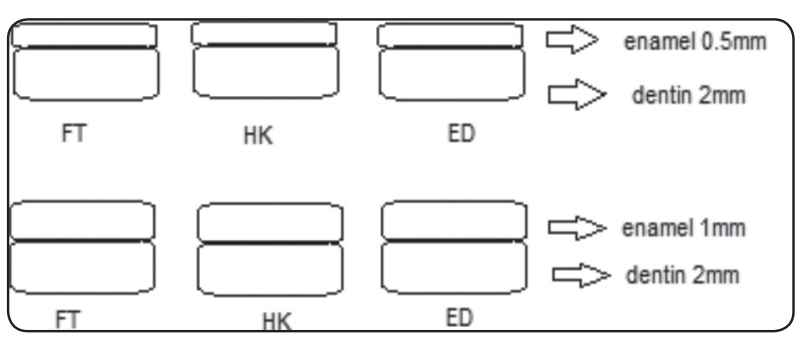

Fig. (1) illustration of sample preparation and thickness of composite resin.

\section{Color measurement:}

Vita easy shade guide spectrophotometer (VITA Easyshade Advance 4.0, Zahnfabrik, Germany) was used for color measurement of each specimen which were performed 3 times. The spectrophotometer was adjusted to the instructions that were given by the manufacturer. ${ }^{9}$ Color measurements were expressed using the CEI L*a*b* color system. $\mathrm{L}$ refers to lightness and is designated 0 to 100 with 0 being white and 100 black. The color axes $\mathrm{a}$ and $\mathrm{b}$ represent red to green and yellow to blue respectively with red and yellow being the positive value and green and blue the negative value. ${ }^{9}$

Since difference of human eye to witness change in color differs between individuals therefore color variation $\Delta \mathrm{E}$ was calculated as follows:

$$
\Delta \mathrm{E}=\sqrt{\left[(\Delta L)^{2}+(\Delta a)^{2}+(\Delta b)^{2}\right.}
$$

Clinical significance was set at $\Delta \mathrm{E} \geq 3.3$ (perceptibility threshold).

The value of each sample is equal to the $\mathrm{L}$ color coordinate, whereas Chroma is calculated using the equation:

$$
c=\sqrt{\left(a^{2}+b^{2}\right.}
$$

\section{Transparency parameter}

The ability of an object to allow the underlying color to show through is referred to as translucency. In this case the outer enamel layer translucency was measured to study its translucency at different thicknesses and its effect on the overall color of the final restoration. ${ }^{10}$

In each specimen the translucency was measured by placing each specimen once against black background and once against white background. Three measurements were taken. Translucency was measured using transparency parameter (TP) using the following formula.

$$
\mathrm{TP}=\left[\left(L_{w}^{*}-L_{B}^{*}\right)^{2}+\left(a_{w}^{*}-a_{B}^{*}\right)^{2}+\left(b_{w}^{*}-b_{B}^{*}\right)^{2}\right]^{2}
$$

The " $w$ " and "B" refer to the value against white and black backings respectively. ${ }^{4}$

\section{Statistical analysis:}

One-way ANOVA and Tukey HSD test were done in order to see if there was any significant difference in TP.

Descriptive statistics were analyzed using the one-way ANOVA, followed by Tukey (HSD) post hoc test.

\section{RESULTS}

The measurement of $\Delta \mathrm{E}$ is only possible by calculating the difference between two shade values so it is deliberated as one set of $\mathrm{L}^{*}, \mathrm{a}^{*}$, and $\mathrm{b}^{*}$ values. Perceptibility threshold of $\Delta \mathrm{E}$ is set at $\Delta \mathrm{E}=3.3 .^{5}$

Group FT showed significant change in color at $\Delta \mathrm{E}=5.32$. Whereas groups $\mathrm{ED}$ and $\mathrm{HK}$ showed no significant change in color at $\triangle \mathrm{E}=2.49$ and $\Delta \mathrm{E}=2.08$ respectively. Using one-way ANOVA and tukey HSD test to evaluate $\Delta \mathrm{E}$ the p-value corresponding to the F-statistic of one-way ANOVA is lower than 0.05 , suggesting that one or more treatments are significantly different. Post hoc comparisons using the Tukey HSD test indicated that the mean score for brand FT was significantly different than the mean score of brands HK and ED (p-value $=0.0388$ $<0.05)$ and ( $\mathrm{p}$-value $=0.0304<0.05)$ respectively. 
Alternatively, comparison between HK and ED showed no significant difference ( $\mathrm{p}$-value $=0.900$ $>0.05)$

Descriptive statistics of $\mathrm{L}^{*}, \mathrm{a}^{*}$, and $\mathrm{b}^{*}$ sample measurement are shown in table 2 . The $\mathrm{L}$ component of the CIELAB is equal to the value of the composite resin. Value is represented in graph 1. L coordinate or value for Brands FT and HK increased as thickness increased unlike brand ED which decreased with increase in thickness. The change in value was studied by one-way ANOVA and tukey HSD test the p-value corresponding to the F-statistic of one-way ANOVA is lower than 0.05 suggesting that all groups showed no significant change in the L coordinate. Thus, the value of color for all groups displayed no significant change as thickness increased.

Table (2) Descriptive statistics of CIE Lab measurement values at enamel thickness 0.5 and $1 \mathrm{~mm}$ with white backing.

\begin{tabular}{|c|c|c|c|c|c|}
\hline \multirow{2}{*}{$\begin{array}{c}\text { Dentin } \\
\text { thickness }\end{array}$} & $\begin{array}{c}\text { Enamel } \\
\text { thickness }\end{array}$ & $\begin{array}{c}\text { Group } \\
\text { code }\end{array}$ & $\begin{array}{c}\mathrm{L} \\
\text { (mean) }\end{array}$ & $\begin{array}{c}\mathrm{a} \\
\text { (mean) }\end{array}$ & $\begin{array}{c}\mathrm{b} \\
\text { (Mean) }\end{array}$ \\
\hline \multirow{3}{*}{$2 \mathrm{~mm}$} & \multirow{3}{*}{0.5} & FT & 92.81 & 0.52 & 25.39 \\
\cline { 3 - 6 } & & HK & 90.61 & 2.69 & 25.30 \\
\cline { 3 - 6 } & \multirow{3}{*}{1} & ED & 92.47 & 1.14 & 29.34 \\
\cline { 3 - 6 } & \multirow{2}{*}{1} & FT & 93.99 & -0.64 & 20.34 \\
\cline { 3 - 6 } & & HK & 92.64 & 2.38 & 25.63 \\
\cline { 3 - 6 } & & ED & 90.49 & 0.43 & 28.01 \\
\hline
\end{tabular}

Table (3) Descriptive statistics of CIE Lab measurement of Chroma and translucency at enamel thickness $0.5 \mathrm{~mm}$ and $1 \mathrm{~mm}$.

\begin{tabular}{|c|c|c|c|c|}
\hline \multirow{2}{*}{$\begin{array}{c}\text { Dentin } \\
\text { thickness }\end{array}$} & $\begin{array}{c}\text { Enamel } \\
\text { thickness }\end{array}$ & $\begin{array}{c}\text { Group } \\
\text { code }\end{array}$ & $\begin{array}{c}\text { Chroma } \\
\text { Mean }\end{array}$ & $\begin{array}{c}\text { Translucency } \\
\text { mean }\end{array}$ \\
\hline \multirow{3}{*}{2} & \multirow{3}{*}{$0.5 \mathrm{~mm}$} & FT & 25.39 & 2.41 \\
\cline { 3 - 5 } & & HK & 25.44 & 4.34 \\
\cline { 3 - 5 } & \multirow{3}{*}{$1 \mathrm{~mm}$} & ED & 29.36 & 5.23 \\
\cline { 3 - 5 } & & FT & 20.35 & 1.49 \\
\cline { 3 - 5 } & & HK & 25.74 & 3.54 \\
\hline \multirow{2}{*}{$*$} & ED & 28.00 & 3.83 \\
\hline
\end{tabular}

Chroma values are found in table 3 and represented in graph 2. Group HK showed an increase in chroma as thickness increase. One-way ANOVA and tukey HSD test were used to analyze the results. The p-value corresponding to the F-statistic of one-way ANOVA is lower than 0.05 this suggests that even though there was an increase in chroma for group HK but the result was insignificant at (p-value $=0.56>0.05$ ). Group FT and ED witnessed a decrease in chroma the results were significant at $(p$-value $=0.00073<0.05)$ and $(p$-value $=0.004<0.05)$ respectively.

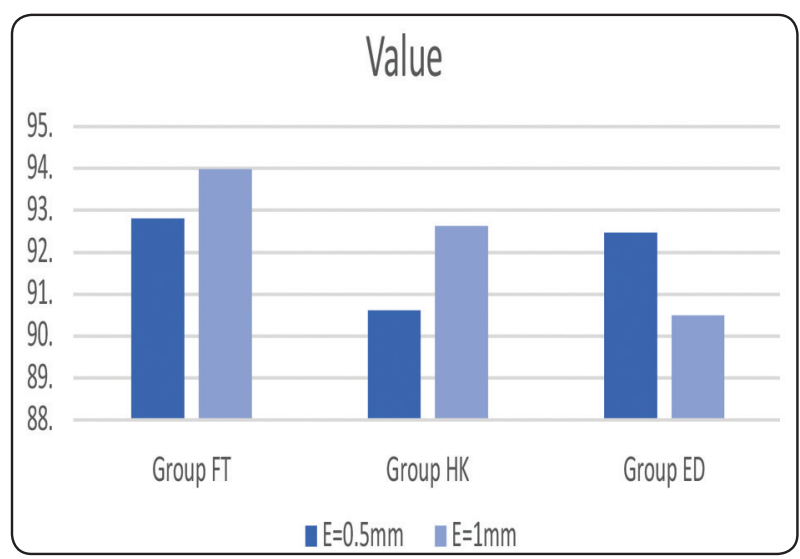

Graph (1) Graph 3: Bar graph representing the change in Value for the three different composite groups FT, HK, and $\mathrm{ED}$ as thickness increases.

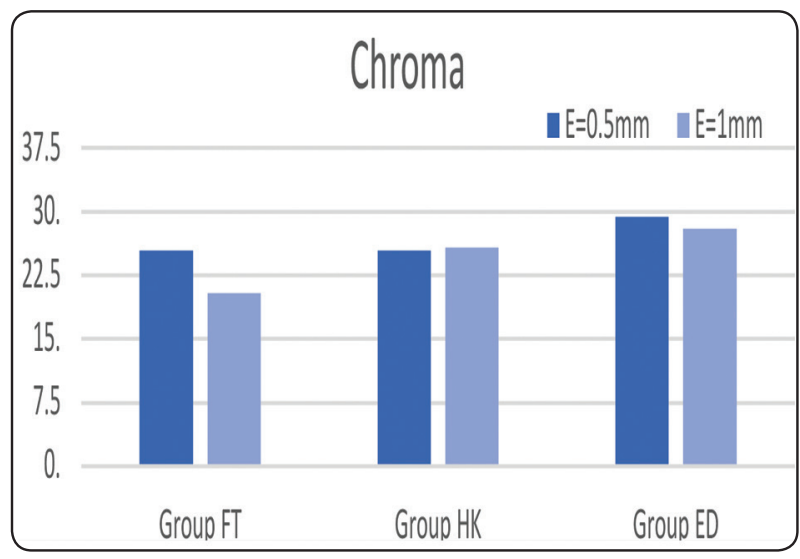

Graph (2) Bar graph representing the change in chroma for the three different composite groups FT, HK, and ED as thickness increases. 


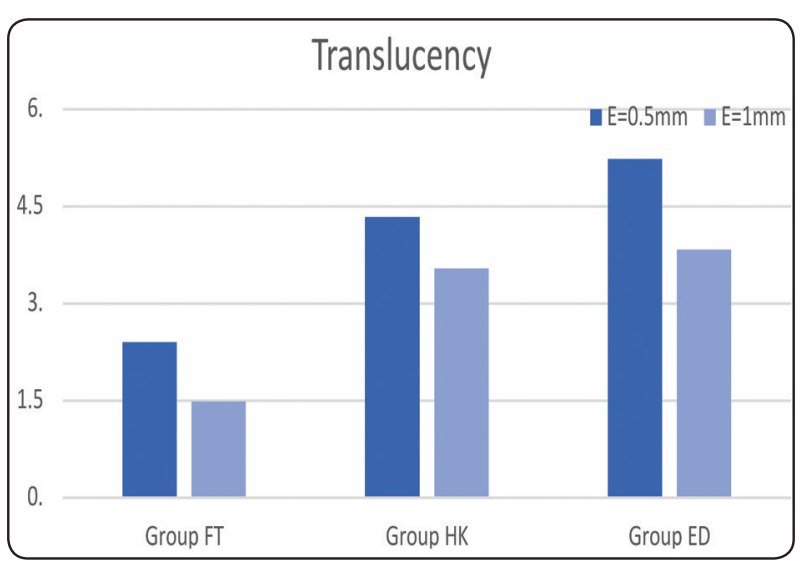

Graph (3) Bar graph representing the change in Translucency for the three different composite groups FT, HK, and ED as thickness increases.

Translucency parameter values showed a decrease in all groups when the enamel thickness increased to $1 \mathrm{~mm}$ the values are observed in table 3 . One-way ANOVA and Tukey HSD test were done in order to see if there was any significant difference in TP.

The p-value corresponding to the F-statistic of one-way ANOVA is lower than 0.05. All groups FT, HK and ED showed a significant change in translucency at $(\mathrm{p}$-value $=0.0399<0.05),(\mathrm{p}$-value $=$ $0.0245<0.05)$, and ( $\mathrm{p}$-value $=0.0004<0.05)$ respectively.

Intergroup analysis showed significant difference in translucency when comparing group FT with $\mathrm{HK}(\mathrm{P}$-value $=0.00101<0.05)$ and ED (p-value $=0.00101<0.05$ at both thicknesses

Unlike HK versus ED which showed no significant difference ( $\mathrm{p}$-value $=0.1147>0.05$ ) these values are at enamel thickness $0.5 \mathrm{~mm}$. At thickness $1 \mathrm{~mm}$ the significance was the same FT versus HK (p-value $=0.00101<0.05$ ) and FT versus ED $(\mathrm{p}$-value $=0.00101<0.05)$ both values show that there is significant difference. ED versus HK (pvalue $=0.6706>0.05$ ) shows that there is no significant difference in translucency between composite brands at same thickness.

\section{DISCUSSION}

In the dental literature, translucency is characterized as partial opacity; or described between complete opacity and complete transparency. ${ }^{15}$ this is vital in choosing esthetic composite resins to restore tooth structure as it affects the relative degree to which materials allow or block any underlying color from affecting the appearance of a colorant layer and in turn significantly affects shade matching. ${ }^{16}$

When a composite restoration is to be placed adjacent to natural teeth, color matching becomes a crucial factor of success. In fact, color becomes the main target and the clinician neglects thickness which plays a major role in color perception of the restoration. The increase in thickness has a direct effect on the final color perception of the restoration. The chroma, translucency, and value are all affected by the change in thickness. ${ }^{5,16}$

Another challenge is encountered when placing restorative materials in large class III or IV where the darkness of the oral cavity affects relatively translucent materials, a grayish effect often occurs. ${ }^{12,13}$

In this study 3 different brands of composite resin were used at two different enamel thickness. The translucency of all composite resins decreased as thickness increased the result was significant at $(\mathrm{p}<0.05)$. Schmeling et al. also came to the same conclusion that as thickness increased the translucency decreased. ${ }^{14}$ A study by kamishima et al also concluded that the increase in thickness decreased the translucency of the composite material but this decrease was even more apparent with enamel shades that showed larger translucency to begin with. Therefore, they deduced that the more translucent the enamel shade that more it is easily affected by the increase in thickness ${ }^{5}$ In the present study group ED showed the highest translucency value. The $p$-value $=0.0004$ was the lowest by far among the groups which showed it had major significant change in translucency. 
Part of the null hypothesis was rejected since the statistical analysis showed that there is a significant change in translucency as thickness increased.

The enamel shade in this study is considered a translucent shade by the manufacturers. The thickness of the outer enamel layer has a direct effect on the overall perception of the color of the restoration ${ }^{9}$. Unlike a study by Kamishima et al which came to the conclusion that enamel had no major effect on the overall color of the restoration. In their study the color difference $(\Delta \mathrm{E})$ between underlying color base and layered resin composite showed no significant difference. ${ }^{5}$ Another study by Lee et al came to the conclusion that the outer enamel shade does have a major effect on the perception of the final color of the restoration. ${ }^{17}$ This study also proved that the increase in thickness had significant change in color $\Delta \mathrm{E}$ of the composite resin, even when comparing intergroup analysis FT compared with $\mathrm{HK}$ and ED there was a perceptible variation in color at $(\mathrm{p}<0.05)$. Unlike the comparison between HK and ED which showed no significant variation.

A study by Schmeling et al showed that the value of the $\mathrm{L}^{*}$ component decreases with the increase of thickness which results in a grayish final color of restoration which is unaesthetic. ${ }^{18}$ In this study the descriptive statistical results did not coincide with Schmeling's conclusion for groups FT and HK the $\mathrm{L}^{*}$ component value increased as thickness increased. Unlike Group ED which witnessed a decrease in $\mathrm{L}^{*}$ component value. But the change in all groups as analyzed by one-way ANOVA and tukey HSD post-hoc test proved to be insignificant. Therefore, the change is not perceptible by the naked eye.

A study by Duarte et al came to the conclusion that as thickness increased the chroma increased, but their study was done using only enamel layer and no dentin background. ${ }^{16}$ Ferraris et al stated that the increase in thickness of enamel layer on dentin background will result in desaturation of the dentin color which will result in decrease in chroma value. ${ }^{4}$ In the present study the results were similar to Ferraris et al, chroma values decreased as thickness increased for groups FT and ED. Group HK witnessed an increase in chroma but this change was insignificant.

Consequently, the whole null hypothesis was rejected since that statistical analysis of chroma revealed that the increase in thickness decreased the chroma value significantly.

\section{CONCLUSION:}

With the limitations of this study the increase in thickness leads to a decrease in chroma and translucency. Our results recomended that extra care and attention must be considered in the thickness of the enamel layer when replicating the translucency of natural adjacent teeth and matching their chroma.

\section{REFERENCES}

1. Dietschi D. Layering concepts in anterior composite restorations. Journal of Adhesive Dentistry. 2001 Mar $1 ; 3(1)$.

2. Sugii, Mari M.Caldas, Ricardo A. Gouvea, Thayla H. N.Nunes Leite Lima, Débora Alves, Marchi, Giselle M. Baggio Aguiar, Flávio Henrique. Utilizing the optical properties of composite resins to improve esthetics: a layering technique for anterior restorations. General Dentistry. Jan/Feb2019, Vol. 67 Issue 1.

3. Vichi A, Fraioli A, Davidson CL, Ferrari M. Influence of thickness on color in multi-layering technique. dental materials. 2007 Dec 1;23(12):1584-9.

4. Ferraris F, Diamantopoulou S, Acunzo R, Alcidi R. Influence of enamel composite thickness on value, chroma and translucency of a high and a nonhigh refractive index resin composite. Int J Esthet Dent. 2014;9(3):382-401

5. Kamishima N, Ikeda T, Sano H. Color and translucency of resin composites for layering techniques. Dental materials journal. 2005;24(3):428-32.

6. Kamishima N, Ikeda T, Sano H. Effect of enamel shades on color of layered resin composites. Dental materials journal. 2006;25(1):26-31. 
7. Khashayar G, Dozic A, Kleverlaan CJ, Feilzer AJ, Roeters $\mathrm{J}$. The influence of varying layer thicknesses on the color predictability of two different composite layering concepts. Dental Materials. 2014 May 1;30(5):493-8.

8. Yu B, Lee YK. Influence of color parameters of resin composites on their translucency. Dent Mater. 2008;24:12361242 .

9. Kim DH, Park SH. Evaluation of resin composite translucency by two different methods. Operative dentistry. 2013 Apr;38(3):E76-90

10. S. H. Q. Quek A. U. J. Yap, V. Rosa , K. B. C. Tan , K. H. Teoh. Effect of staining beverages on color and translucency of CAD/CAM composites. Journal of Esthetic \& Restorative Dentistry. Mar2018, Vol. 30 Issue 2, pE9-E17.

11. Darabi, Farideh; Radafshar, Golpar; Tavangar, Marjan; Davaloo, Reza; Khosravian, Aref; Mirfarhadi, Nastaran. Translucency and Masking Ability of Various Composite Resins at Different Thicknesses . Journal of Dentistry (17283426). Sep2014, Vol. 15 Issue 3, p117-122.

12. Sidhu SK, Ikeda T, Omata Y, et al. Change of color and translucency by light curing in resin composites. Oper Dent 2006;31(5):598-603.
13. Inokoshi S, Burrow MF, Kataumi M, et al. Opacity and color changes of tooth-colored restorative materials. Oper Dent 1996;21:73-80.

14. Schmeling, M., DE ANDRADA, M.A.C., Maia, H.P. and De Araujo, E.M. Translucency of value resin composites used to replace enamel in stratified composite restoration techniques. Journal of Esthetic and Restorative Dentistry, 2012. 24(1), pp.53-58.

15. Johnston WM, Reisbick MH. Color and translucency changes during and after curing of esthetic restorative materials. Dent Mater 1997;13:89-97.

16. Duarte Jr, S., de Oliveira, M., Botta, A.L. and Phark, J.H., 2011. Influence of Enamel Layering Thickness on Chroma, Value, and VITAShade for Esthetic Composite Resin Restorations. American Journal of Esthetic Dentistry, 1(2).

17. Lee, Y.K. and Powers, J.M., 2004. Calculation of colour resulting from composite/compomer layering techniques. Journal of oral rehabilitation, 31(11), pp.11021108.

18. Schmeling, M., Meyer-Filho, A., Andrada, M.A.C. and Baratieri, L.N., 2010. Chromatic influence of value resin composites. Operative dentistry, 35(1), pp.44-49. 